\title{
Power Optimized Routing in Mobile Ad Hoc Networks
}

\author{
Ramanna Havinal, Girish V. Attimarad and M.N. Giriprasad
}

\begin{abstract}
We are moving from the Personal Computer (PC) age (i.e., one computing device per person) to the Ubiquitous Computing age in which individual users utilize, at the same time, several electronic platforms through which they can access all the required information whenever and wherever they may be. Since the basic components of ad hoc wireless networks are mostly battery-operated portable devices, power conservation is one of the central issues of such networks. Power-conservative designs for ad hoc networks pose many challenges due to the lack of central coordination facilities. The purpose of this paper is to provide a comprehensive discussion of the power-optimization in mobile ad hoc networks based on routing.
\end{abstract}

Keywords--- Energy Efficiency, On Demand.

\section{INTODUCTION}

$\mathrm{W}$ IRELESS data networks are increasingly becoming an important part of the next-generation network infrastructure. This is made possible by the availability of inexpensive wireless network devices such as Bluetooth and wireless LANs. The objective of these networks is to provide users with "anytime, anywhere" data access. The end-user devices range from small handheld PDAs to larger laptops. The computing and storage capabilities of these devices cover a wide spectrum. One of the main limitations of these wireless networks is the limited battery power of the network nodes. Therefore, power management is one of the challenging problems in wireless communication, and recent research has addressed this problem. Wireless networks are typically classified as: (i) infrastructure networks, in which all end node communication is through a more powerful entity called the base station, which is connected to a wired network infrastructure; and (ii) ad hoc networks, in which end nodes establish a network among themselves and communicate with each other in a multihop manner.

Specifically,advances in wireless communication will enable a radical new communication paradigm: self-organized information and communication systems. In this new networking paradigm, the users' mobile devices are the network, and they must cooperatively provide the functionality that is usually provided by the network

Ramanna Havinal, Associate Professor, ECE Department, College of Engg Ambajogai, India. E-mail:rshavinal@gmail.com

Girish V. Attimarad, Head of ECE Department, KSSEM Bengaluru, India.E-mail:gattimarad@yahoo.com

M.N. girirasad Professor ECE, Department, JNTUA College Of Engg AnanthapurIndia.E-mail:mahandragiri1960@gmail.com

DOI:10.9756/BIJSESC.8245 infrastructure (e.g., routers, switches, and servers). Such systems are sometimes referred to as mobile ad hoc networks (MANETs) or as infrastructure-less wireless networks

Routing is a significant consumer of battery power since a packet is routed through many intermediate nodes before reaching its destination. Energy costs related to communication can be high in mobile nodes but this chapter only considers the costs related to routing. The design of energy-efficient routing protocols has attracted the attention of researchers in the past few years

\section{A. Sources of Power Consumption}

The sources of power consumption, with regard to network operations, can be classified into two types: communicationrelated and computation-related. Communication involves usage of the transceiver at the source, intermediate (in the case of ad hoc networks), and destination nodes. The transmitter is used for sending control, route request, and response messages, as well as data packets originating at or routed through the transmitting node. The receiver is used to receive data and control packets, some of which are destined for the receiving node and some of which are forwarded. Understanding the power characteristics of the mobile radio used in wireless devices is important for the efficient design of communication protocols. A typical mobile radio may exist in three modes: transmit, receive, and standby. Maximum power is consumed in the transmit mode, and the least in the standby mode. Thus, the goal of protocol development for environments with limited power resources is to optimize the transceiver usage for a given communication task. Computation costs, involving packet processing and the CPU, are not considered in this chapter.

Battery technology has lagged compared to the advancements in communication and computing technology in the past decade. Now that batteries' capacity cannot be significantly improved, efforts should be put into designing energy-efficient software and hardware. A portable device typically has several main hardware components that consume battery power: display monitor, disk, CPU, memory, and wireless network interface card (WNIC). The WNIC component can consume $10-50 \%$ of overall system energy, which explains why notebooks' lifetimes reduce significantly when inserted with WNICs.

Wireless architectures can be classified as Infrastructure and ad hoc. With infrastructure (base stations), energyefficient techniques are usually easier to develop due to the availability of central coordination. Without such central coordination, power-conservative designs pose more challenges for ad hoc networks. We generally divide powerconservative protocols into two categories: 
1. Transmitter Power control mechanisms

2. Power management algorithms

The latter can be further classified into MAC-layer (where MAC stands for Medium Access Control), network-layer, and higher-layer implementations.

In wireless ad hoc networks, the nonexistence of a centralized authority complicates the problem of medium access control.Mobile stations may contend simultaneously for medium access. Consequently, transmissions of packets from distinct mobile terminals are more prone to overlap, resulting in packet collision $\mathrm{s}$ and energy losses. Likewise, the performance of the MAC scheme has a great effect on the performance of the routing method employed and on the energy consumption of the wireless network interface card (NIC).

\section{POWER MANAGEMENT IN MANET}

\section{A. MAC-Layer Power Management}

Based on the OSI model, the MAC layer is a sublayer of the data link layer right above the physical layer. The MAC layer is mainly responsible for access scheduling of shared medium. A device's power consumption strongly depends on the MAC policy adopted. Power-efficient designs are even more challenging in ad hoc networks, which lack central coordination. Below, we present several existing MAC-layer power-conservative designs for ad hoc networks.

A Power-Aware Multi-Access protocol with Signalling (PAMAS) is introduced for power conservation in ad hoc networks. PAMAS is a RTS/CTS-based M AC protocol with separate data and signalling channels for data and control packets, respectively. The signalling channel is used for exchanging RTS/ CTS packets, busy tones, and probe control packets. The ba sic observation is that, due to the broadcast nature of radio transmission, a host's energy is often wasted on overhearing packets that are not destined for it. In PAMAS, battery power is conserved by judiciously turning off hosts' radios when no transmission/reception is possible.

The separate signalling channel enables a host to determine when and for how long to power its antenna off. It is easy for a host to decide its power-off duration if RTS/CTS packets can be correctly received. However, if a host misses some RTS/CTS packets during its power-off period, PAMAS provides a probe protocol to estimate the duration of others' remaining transmissions. The ultimate goal is to reduce power consumption without increasing delay and decreasing throughput. Simulation results indicate 10 to $60 \%$ power savings in most cases.

\section{B. Network-Layer Power Management}

We review several power-saving techniques implemented in the network layer for ad hoc networks. Residing above the data link layer, the network layer is responsible for routing packets toward destinations. For wired environments, routing paths are usually fixed since end terminals are static. However, in wireless networks, mobile hosts are free to move, resulting in frequently changed routing paths. The wireless network layer hence needs to take care of mobility management, such as location tracking/update, which is not considered in static wired networks. In an ad hoc network, a packet typically needs to be relayed by several hosts before reaching its final destination. Network-layer power-aware routing is thus a unique issue for ad hoc networks. Traditional ad hoc routing protocols adopt metrics, such as hop-count, link quality, and location stability, for route selection. These metrics, however, do not take battery power conditions into account, which may lead to improper energy expenditure and thus reduced network lifetime.

\section{a) Unicast Data Communications}

Several power-aware routing protocols have been proposed for unicast communication .There are five metrics for power-aware routing. The underlying MAC protocol is PAMAS, which was reviewed earlier in Section 4.3. This work can further improve power consumption by 5-30\% over traditional shortest-path routing protocols. The five metrics are:

1. Minimize energy consumed per packet: Obviously, by minimizing energy consumption for each packet, the total power consumption will be reduced. However, to conserve power, this metric tends to select routes around congested are as. How to share energy cost among hosts is left unaddressed. So the network lifetime may not be improved.

2. Maximize time to network partition : Given an ad hoc network, there exists a minimal set of hosts whose removal will partition the network. The set is called the cut-set. Energies of the hosts in the cut-set are critical and decisive for network lifetime. This metric attempts to divide network load among the cut-set hosts to prolong the time before the network becomes partitioned. However, this optimization problem is similar to a load-balancing problem, which is known to be NP complete.

3. Minimize the variance of hosts' power levels : The goal of this metric is to make all hosts process about the same number of packets to achieve an equal power draining rate at each host. The basic idea is that each host sends packets through a neighbor with the least amount of data waiting to be transmitted.

4. Minimize cost per packet: This metric avoids selecting hosts with depleted battery energy on routing paths. It is possible that delay and energy consumption per packet will be increased. Nevertheless, this is not necessarily a drawback because lifetime variation of hosts is reduced, resulting in longer network lifetime.

5. Minimize maximum node cost: The goal is $\mathrm{t} \mathrm{o}$ minimize the cost paid by a host relaying a packet. Using the metric, the time of first host failure is expected to be delayed. Unfortunately, this metric is an ideal case and not implementable.

In short, power-aware routing should be designed based on shortest-cost instead of shortest-hop metrics, where "cost" can be defined differently but should be energy related 


\section{HIGHER-LAYER POWER MANAGEMENT}

\section{A. Transport Layer}

Residing above the network layer, the transport layer provides end-to-end communication functionality. The most widely adopted transport protocol is TCP (Transmission Control Protocol). Unfortunately, TCP was originally designed for reliable wired environments, where packet error/loss due to interference/fading/ handoff is scarce. When packet losses do happen, TCP will interpret this as channel congestion and invoke congestion control to reduce the transmission rate. This misinterpretation results in reduced throughput and unacceptable delays. Furthermore, because of the bursty-error characteristic of wireless links, transmissions under bad conditions are probably continuing to be lost. This introduces many unnecessary retransmissions and energy was te, but it can be avoided by queuing data for later delivery. Energy-efficient transport protocols attempt to reduce delays and the number of unnecessary retransmissions, both of which are energy consuming.

\section{ROUTING AND ENERGY EFFICIENCY IN MANETS}

Routing protocols in wireless mobile ad hoc networks can be classified as table-driven and on-demand. In table-driven routing protocols, all the mobile stations are required to have complete knowledge of the network through their periodic and incremental/triggered updates. Unnecessary periodic updates may have a negative effect on energy conservation. Tabledriven routing is also known as proactive routing. Examples of table-driven routing protocols include global state routing (GSR) and destination sequenced distance vector (DSDV). Nodes running GSR exchange link states packets (LSPs) with their neighbours. In DSDV, sequence numbers are used to provide a means to enable mobile nodes to distinguish old routes, as a result of mobility, from new ones. Thus, the formation of loops is avoided. On-demand routing requires that routes be built between nodes only as desired by source nodes. Hence, the terms on-demand and reactive can be used interchangeably. On-demand routing has two major components: route discovery and route maintenance. In route discovery, a source uses flooding to acquire a route to its destination. This degrades system-wide energy conservation. The transit nodes, upon receiving a query, learn the path to the source and enter the route in their forwarding tables. The destination node responds using the path traversed by the query. Route maintenance is responsible for reacting to topological changes in the network, and its implementation differs from one algorithm to the other.

On-demand protocols include ad hoc on demand distance vector routing (AODV) and dynamic source routing (DSR). In on-demand protocols, route discovery and maintenance may become inefficient under heavy network load since intermediate nodes will have a higher probability of moving due to the delay in packet transmissions attributed to MAC contention. Hence, routes will also have a higher probability of breaking as a result of mobility. This wastes battery power, and thus the lifetime of the wireless nodes decreases. Moreover, flooding of route request and route reply packets in on-demand routing protocols may result in considerable energy drains under a realistic energy consumption model that takes idle time and promiscuous mode power into account. Every station that hears the route request broadcasts will consume an amount of energy proportional to the size of the broadcast packet. In addition, stations that hear a corrupted version of a broadcast packet will still consume some amount of energy. Besides, the simulation studies carried out for tabledriven and on-demand routing protocols fall short of providing necessary power-based performance metrics, such as average node and network lifetime, average dissipated energy per protocol, standard deviation of the energy dissipated by each individual node, etc.

A large number of routing protocols have been developed for mobile ad hoc networks(MANETs) [14], which are characterize $\mathrm{d}$ by unpredictable network topology changes, high degree of mobility, energy-constrained mobile nodes, bandwidth-constrained intermittent connection, and memory constrained. The routing problem has been well researched in infrastructured wireless networks, where the goals are efficient detection and adaptation to the network topology, scalability, and convergence. Even though these are equally valid for MANETs, the solutions are more difficult to find since MANETs are inherently more dynamic. In particular, energy efficiency may be the most important design criterion for mobile networks since a critical limiting factor for a mobile node is its operation time, restricted by battery capacity. In infrastructured wireless networks, where a wireless link is limited to one hop between an energy-rich base station and a mobile node, the goal of energy conservation can be largely achieved by relocating power intensive network operations to the base station.

However, the wireless link -only routing path in a MANET makes energy savings difficult to achieve. The corresponding reduction of nodes' lifetime directly affects the network lifetime since mobile nodes themselves collectively form a network infrastructure for routing in a MANET. To address this problem, many research efforts have been devoted to developing energy aware network protocols such as power saving MAC (medium access control) layer protocols, energy efficient routing algorithms, and power sensitive network architectures. Based on the aforementioned discussion, this chapter focuses on the energy-aware routing mechanisms proposed for MANETs.

\section{ROUTING PROTOCOLS FOR ADHOC NETWORKS}

The routing protocols proposed for MANETs are generally categorized as table-driven, source-initiated on-demand driven, and hybrid based on the timing when the routes are updated. With the table-driven routing protocols, each node attempts to maintain consistent, up-to-date routing information to every other node in the network. With source-initiated ondemand routing, route discovery and maintenance are performed only when a source node desires them. The hybrid approac $\mathrm{h}$ combines the two approaches to minimize the overhead incurred during route discovery and maintenance. In this section, the protocols belonging to each of the three aforementioned categories are discussed. 


\section{A. Table-Driven Routing Protocols}

In table-driven routing protocols, each node maintains an up-to-date routing table by responding to changes in network topology and propagating the updates. Thus, it is proactive in the sense that when a packet needs to be forwarded, the route is already known and can be immediately used. As is the case for wired networks, each node in a MANET main tains a routing table containing a list of all the destinations, next hop, and the number of hops to each destination. The routing table is constructed using either link-state or distance vector algorithms. There are a number of protocols that belong to this category, which are different in the number of tables manipulated for routing and the methods used for exchanging and maintaining routing tables. Among the table-driven protocols, Destination-Sequenced Distance Vector (DSDV), Wireless Routing Protocol (WRP), and Global State Routing (GSR) use destination sequence numbers to keep routes loop free and up to date. These sequence num bers are assigned by the destination node and allow the mobile nodes to distinguish invalid routes from new ones. GSR is similar to the DSDV scheme but uses the link state instead of the distance vector. Ea ch node maintains a link-state table based on the information exchanged periodically with the neighb ors. The update is selected based on the timestamp of the sequence numbers. In WRP, each node mainta ins a distance table, a routing table, a link-cost table, and a Message Retransmission List (MRL) table. MR L keeps a record of which updates in an update message need to be retransmitted and which neighbors should acknowledge the retransmission. An update message is sent only between neighboring no des and contains a list of updates (the destination, the distance to the destination, and the predecesso $\mathrm{r}$ of the destination), as well as a list of responses indicating which mobile nodes should acknowledge (ACK) the update. In contrast to DSDV and GSR, Cluster Gateway Switching Routing (CGSR) [6], Hierarchical State Routing (HSR), and Zone-based Hierarchy Link State (ZHLS) protocols use hierarchical routing schemes. The CGSR protocol extends DSDV by grouping nodes into clusters. Thus, each cluster is represented by a cluster head, and two clusters can communicate via a gateway node that is within the communication range of the two clusters. Each nod e also maintains a cluster member table where the cluster heads' destinations are stored. Therefore, the cluster member table is used to perform inter cluster routing, while the routing table is used to perform in intra cluster routing. The HSR protocol extends CGSR by forming a hierarchy of cluster heads. This is don e by having nodes within a cluster broadcast their link information to each other. The cluster head summarizes its cluster's information and sends it to neighboring cluster heads via gateway as done in CG SR. The hierarchy reduces the overhead associated with the link-state algorithm and the number of entries in the routing table. In ZHLS, the network is divided into non overlapping zones without any zone-head . ZHLS defines two levels of topologies - node level and zone level. If any two nodes are within the communication range, a physical link exists. A virtual link exists between tw o zones if at least one node of a zone is physically connected to some nodes of the other zone. The no de (zone) level topology provides the information on how the nodes (zones) are connected together b y the physical (virtual) links. Thus, given the zone and node ID of a destination, the packet is routed based on the zone ID until it reaches the correct zone. Then, within that zone, it is routed based on node ID. Fisheye State Routing (FSR) protocol is another hierarchical routing scheme where information exchange is more frequent with closer nodes than with faraway nodes. FSR is an improvement over GSR in which the bandwidth overhead due to update messages is minimized. The FSR protocol scales well to large networks since the overhead is controlled.

\section{B. Source-Initiated On-Demand Driven Protocols}

These are reactive protocols where routes are created only when desire $\mathrm{d}$ by the source node. The two basic procedures of source-initiated on-demand driven protocols are the route discovery process and the route maintenance process. The route discovery process involves sending route-request packets to neighbour nodes, which then forward the request to their neighbors, and so on. On ce the route-request reaches the destination or the intermediate node with a "fresh enough" route, the destination/intermediate node responds by unicasting a route-reply packet back to the neighbor from which it first received the route request.

Once the route is established, it is maintained by some form of route maintenance process until either the destination becomes inaccessible along any path from the source or the route is no longer desired. In contra st to table-driven routing protocols, not all up-to-date routes are maintained at every node. This subsection discusses several source-initiated ondemand routing protocols. The Dynamic Source Routing (DSR): (DSR) protocol is a typical example of the ondemand protocols, where each data $\mathrm{p}$ acket carries in its header the complete ordered list of nodes the packet passes through. This is done by having each node maintain a route cache that learns and caches routes to destinations. Some ondemand routing protocols are extensions of table-driven protocols. For example, the Ad-Hoc On-Demand Vector $(A O D V)$ protocol is an improvement on the DSDV protocol, where the number of required broad casts is minimized by creating routes on an on-demand basis. Each node maintains its own sequence number, as well as a broadcast ID for the route-request. The broadcast ID is incremented for every route-re quest the node initiates, and together with the node's IP address it uniquely identifies a route-request. The Cluster Based Routing Protocol (CBRP )is an extension of CGSR where nodes are divided into clusters. When a source has data to send, it floods route request packets only to the neighboring cluster heads. Upon receiving the request, a cluster head checks to see if the destination is in its cluster. If so, the request is sent directly to the destination; otherwise, the request is sent to all its adjacent cluster heads. Temporally Ordered Routing (TORA) is a highly adaptive protocol that provides multiple routes for any desired so urce-destination pair and localizes the control messages to a very small set of nodes near the location of a topological change. To accomplish this, nodes maintain routing information on adjacent (one-hop) nodes and use a "height" metric to establish a directed acyclic graph (DAG) rooted at the destination. When the DAG route is broken during node 
mobility, route maintenance is necessary to re establish a DAG rooted at the same destination. This is achieved using a link reversal algorithm at the site of the link failure to re establish the path. The algorithm tries to localize the effect and gives many alternate paths to the destination. Thus, the algorithms not only save bandwidth in updates, but also provide alternate paths in case of path failures.

In contrast to aforementioned protocols that only use the shortest path as the routing metric, the Associativity Based Routing $(A B R)$ protocol uses the connection stability metric, called associativity, among mobile no des to select the best route. In other words, a high degree of associativity may indicate a low state of nod e mobility, while a low degree may indicate a high state of node mobility. Associativity among nodes is determined by first having all nodes generate periodic beacons, and then the associativity tick of the receiving node with respect to the beaconing node is incremented. Thus, when packets arrive at the destination, the best route is selected by examining the associativity ticks along each of the paths. Associativity ticks are reset when the neighbors of the node or the node itself move out of proximity. Similarly, the Signal Stability Routing(SSR) protocol selects routes based on signal strength. SSR selects routes bas ed on the signal strength between nodes and on a node's location stability, and it is divided into two cooperative protocols: the Dynamic Routing Protocol (DRP) and the Static Routing Protocol (SRP). DRP is responsible for maintaining the Signal Stability Table (SST) and the Routing Table (RT). SST record s the signal strength of neighboring nodes as strong or weak using periodic beacons from each neighboring node. DRP passes a received packet to the SRP, which then forwards it using the RT. If there is no known route in RT, a route search is initiated by sending route-requests over only strong channels. The destination chooses the first arriving route-request packet to send back because it is most probable that the packet arrived over the shortest and/or least congested path. If no route-reply message is received by the source within a specific timeout period, the source node indicates that weak channels are acceptable, as these may be the only links over which the packet can be propagated. The Relative Distance Micro-Discovery Routing (RDMAR) protocol improves the ABR protocol by limiting the flooding of route-request packets to a certain radius. The estimate of the radius is based on the number of radio hops between two nodes. This protocol does not employ beaconing or a route cache

\section{Hybrid Routing Protocols}

The hybrid approach combines the table-driven and source-initiated on-demand driven approaches such that the overhead incurred in route discovery and maintenance is minimized while the efficiency is maximized. Several protocols belonging to this approach are presented in this subsection .

The Zone Routing Protocol (ZRP) partitions the network implicitly into zones, where a zone of anode includes all nearby nodes within the zo ne radius defined in hops. It applies proactive strategy inside the zone and reactive strategy outside the local zone. Each node may potentially be located in many zones. ZRP consists of two sub protocols. The proactive intra zone routing protocol (IARP) is an adapted distance-vector algorithm. When a source has no IARP route to a destination, it invokes a reactive inter zone routing protocol (IERP), which is very similar to DSR.

The Core Extraction Distributed Ad Hoc Routing (CEDAR) protocol is a hierarchical protocol that attempts to model the IP routing structure, with emphasis on QoS support, by identifying a subset of nodes called core nodes. Each node must be adjacent to at least one core node and picks one node as the leader or dominator. The core is determined by periodic exchange of messages between each node and its neighbors. Each core node maintains a path to the nearby nodes by issuing a limited broadcast. The core is dynamically extracted by approximating a minimum dominating set using local computation and local state, and it performs route computation on behalf of the nodes that belong to it. The bandwidth availability information is then propagated $\mathrm{i} n$ the core subgraph. Each core node knows local links and nodes that are stable or having high bandwidth. When a source wants to send a packet to the destination, it informs its core. The core node then find $s$ the path to the core node of the destination using some DSR-like probing. Finally, core nodes form a path using locally available link-state information.

The Location-Aided Routing (LAR) protocol assumes that the sender has advance knowledge of the location and velocity of the destination node using the GPS. Based on the location and velocity of the destination node, the expected zone can be defined. Thus, LAR limits the search for a new route to a small zone resulting in fewer route discovery messages. The request zone is the smallest rectangle that encompasses the expected zone. The sender explicitly specifies the request zone in its route-request message to limit the boundary on the propagation of the route-request messages.

The Distance Routing Effect Algorithm for Mobility (DREAM) protocol uses the fact that the greater the distance separating two nodes, the slow er they appear to be moving with respect to each other. Accordingly, the location information in routing tables can be updated as a function of the distance separating the nodes without compromising the routing accuracy. DREAM sends the location updates by the moving nodes autonomously, based only on the node's mobility rate. This is because routing information on the slowly moving nodes needs to be updated less frequently than that for those with high mobility. This is done by sending messages in the "record direction" of the destination node, guaranteeing delivery by following the direction with a given probability.

\section{CONCLUSION}

Considering today's portable/wireless device s, batteries are essential components. If battery power is not used efficiently, these devices may soon bec ome burdens to us once their energy is drained out, no matter how fantastic the applications/services they can provide. In the near future, ad hoc networks are very likely to become popular due to their flexibility. With similar advantages, sensor networks, also in the form of ad hoc networks, are gaining more and more attention as well. Since small sensors are also battery operated, 
power conserv ation is bound to be a major issue for sensor networks

\section{REFERENCES}

[1] M. Ilyas, "The handbook of ad hoc wireless networks", CRC press, 2002.

[2] M.M. Artimy, W. Robertson and W.J. Phillips, "Algorithms and Protocols for Wireless and Mobile Ad Hoc Networks", 2009.

[3] I. Stojmenovic, "Handbook of wireless networks and mobile computing", Vol. 27, 2003.

[4] C.E. Jones, K.M. Sivalingam, P. Agrawal and J.C. Chen, "A survey of energy efficient network protocols for wireless networks", ACM/Baltzer Wireless Networks, Vol. 7, No. 4, Pp. 343-358, 2001.

[5] J.P. Macker and M.S. Corson, "Mobile ad hoc networking and the IETF", ACM SIGMOBILE Mobile Computing and Communications Review, Vol. 2, No. 1, Pp. 9-14, 1998.

[6] G. Pottie, and W. Kaiser, "Wireless integrated network sensors", Communications of the ACM, Vol. 43, No. 5, Pp. 51-58, 2000.

[7] A. Salkintzis and P.T. Mathiopoulos, "The evolution of mobile data networking”, IEEE Personal Communications, Vol. 7, No. 2, Pp. 4-7, 2000.

[8] M. Srivastava, "Tutorial: Energy efficiency in mobile computing and networking", ACM Mobi-Com Tutorials, 2000.

[9] R. Kravets and P. Krishnan, "Power management techniques for mobile communication", In Proceedings of the 4th annual ACM/IEEE international conference on Mobile computing and networking, Pp. 157$168,1998$.

[10] R. Kravets, K. Schwan and K. Calvert, "Power-aware communication for mobile computers", In Mobile Multimedia Communications, 1999.(MoMuC'99) 1999 IEEE International Workshop on, Pp. 64-73, 1999.

[11] M. Zorzi and R.R. Rao, "Energy efficiency of TCP in a local wireless environment", Mobile Networks and Applications, Vol. 6, No. 3, Pp. 265-278, 2001.

[12] T. Imielinski and H. Korth, "Kluwer Academic Publishers", Dordrecht, Pp. 153-181, 1996.

[13] S. Murthy and J.J. Garcia-Luna-Aceves, "An efficient routing protocol for wireless networks", Mobile Networks and applications, Vol. 1, No. 2, Pp. 183-197, 1996. 